\title{
Four new species of the primitively segmented spider genus Qiongthela from Hainan island, China (Mesothelae, Liphistiidae)
}

\author{
Xin $\mathrm{Xu}^{1,2}$, Fengxiang Liư ${ }^{2}$, Matjaž Kuntner ${ }^{2,3,4}$, Daiqin $\mathrm{Li}^{5}$ \\ I College of Life Sciences, Hunan Normal University, Changsha, Hunan, China 2 Centre for Behavioural \\ Ecology and Evolution (CBEE), College of Life Sciences, Hubei University, Wuhan, Hubei, China 3 Institute \\ of Biology, Research Centre of the Slovenian Academy of Sciences and Arts, Ljubljana, Slovenia 4 Department \\ of Entomology, National Museum of Natural History, Smithsonian Institution, Washington, D. C, USA 5 De- \\ partment of Biological Sciences, National University of Singapore, 14 Science Drive 4, Singapore 117543 \\ Corresponding author: Xin Xu (xuxin_09@163.com); Daiqin Li (dbslidq@nus.edu.sg)
}

Academic editor: J. Miller | Received 27 July 2017 | Accepted 19 October 2017 | Published 6 November 2017

http://zoobank.org/9D61D30E-91C0-4A5E-8830-4D10A8DA9553

Citation: Xu X, Liu F, Kuntner M, Li D (2017) Four new species of the primitively segmented spider genus Qiongthela from Hainan island, China (Mesothelae, Liphistiidae). ZooKeys 714: 1-11. https://doi.org/10.3897/zookeys.714.19858

\begin{abstract}
Four species of the primitively segmented spider genus Qiongthela Xu \& Kuntner, 2015 collected from Hainan Island, China are diagnosed and described as new to science based on their genital morphology:

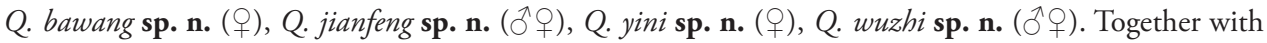
the type species of Qiongthela, Q. baishensis Xu, 2015, five species are found and described from Hainan, China, and seven species are now known in the genus.
\end{abstract}

\section{Keywords}

China, primitively segmented spiders, Qiongthela, taxonomy, trapdoor spiders 


\section{Introduction}

The primitively segmented spider family Liphistiidae (Araneae, Mesothelae), an ancient lineage that retains plesiomorphic arachnid traits such as the abdominal tergites and spinnerets located in the middle of abdominal venter, currently contains 101 species in eight genera (World Spider Catalog 2017; Xu et al. 2015a, b). These extant species are restricted to Southeast and East Asia and display an extremely high level of endemism and disjunct distribution (Xu et al. 2015a, b, 2016). They are divided into two subfamilies, Heptathelinae and Liphistiinae (Xu et al. 2015a, b). While the subfamily Liphistiinae contains a single genus (Liphistius Schiödte, 1849) with species in Southeast Asia (Sumatra, Laos, Malaysia, Myanmar, Thailand), Heptathelinae has seven genera. Two of them (Heptathela Kishida, 1923 and Ryuthela Haupt, 1983) are confined to Japan (Ryukus islands and Kyushu), and the other five are distributed between mainland China and Vietnam: Ganthela Xu \& Kuntner, 2015, Qiongthela Xu \& Kuntner, 2015, Sinothela Haupt, 2003, Songthela Ono, 2000 and Vinathela Ono, 2000) (World Spider Catalog 2017; Xu et al. 2015a, b). Since the genus level revision of the family (Xu et al. 2015a, b), species-level revisions have been proposed for two genera, Ganthela (Xu et al. 2015c) and Ryuthela (Xu et al. 2017).

In this study, we focus on the genus Qiongthela from Hainan Island, China, located in the transitional zone between tropical and temperate zones in the South China sea. Qiongthela was diagnosed in 2015 (Xu et al. 2015a, b), but so far, only three Qiongthela species have been named: Q. baishensis $\mathrm{Xu}, 2015$, the type species from Hainan, and Q. australis (Ono, 2002) and Q. nui (Schwendinger \& Ono, 2011) from southern Vietnam (Xu et al. 2015b). Even though no molecular data were available for the two Vietnamese species of Qiongthela in our previous studies (Xu et al. 2015a), the genital morphology confirms their inclusion in Qiongthela (Ono 2002; Schwendinger and Ono 2011; Xu et al. 2015b). Here, four new species of Qiongthela collected from Hainan are diagnosed and described. Their taxonomy is based on male and female genital morphology combined with the results from prior phylogenetic analyses, which support the four new species (fig. 2 in Xu et al. 2015a).

\section{Materials and methods}

All Qiongthela specimens in this study were collected at the roadside of forest (Figs 1A, $2 \mathrm{~A}, 4 \mathrm{~A})$. They were collected alive and fixed in absolute alcohol if they were adults. The subadults were brought back to the laboratory and reared until they reached maturation. All specimens were then preserved in $80 \%$ ethanol after the right four legs were removed for molecular work.

Specimens were studied using an Olympus SZX16 stereomicroscope. Anatomical details were examined and photographed with on Olympus BX53 compound microscope and a Canon 7D camera. Genitalia were cleared in boiling $\mathrm{KOH}$ for a few minutes to dissolve soft tissues. All the specimens were deposited at the Centre for Behavioural 
Ecology and Evolution (CBEE), College of Life Sciences, Hubei University, Wuhan, China. All lengths are given in millimetres. Leg and palp measurements are given in the following order: total length (femur + patella + tibia + metatarsus + tarsus).

Abbreviations used:

$\begin{array}{llll}\text { ALE } & \text { anterior lateral eyes, } & \text { E } & \text { embolus, } \\ \text { AME } & \text { anterior median eyes, } & \text { OL } & \text { opisthosoma length, } \\ \text { BL } & \text { body length, } & \text { OW } & \text { opisthosoma width, } \\ \text { CL } & \text { carapace length, } & \text { PLE } & \text { posterior lateral eyes, } \\ \text { Co } & \text { conductor, } & \text { PME } & \text { posterior median eyes, } \\ \text { CT } & \text { contrategulum, } & \text { RC } & \text { receptacular cluster, } \\ \text { CW } & \text { carapace width, } & \text { T } & \text { tegulum. }\end{array}$

\section{Taxonomy}

\section{Genus Qiongthela Xu \& Kuntner, 2015}

Type species. Qiongthela baishensis Xu, 2015

Diagnosis. Qiongthela males can be distinguished from all other Heptathelinae genera by the blade-like conductor narrowing to a slightly hooked apex (Fig. 2M-O; Fig. 4F-J), and by the tegulum with two distinct apophyses (Fig. 2L-O; Fig. 4F-J). The females differ from all other Heptathelinae genera by two paired receptacular clusters, all with numerous granula (e.g. Fig. 1C-H) (Xu et al. 2015b).

Species composition. Q. australis (Ono, 2002), Q. nui (Schwendinger \& Ono, 2011), Q. baishensis Xu, 2015, Q. bawang sp. n., Q. jianfeng sp. n., Q. yini sp. n., Q. wuzhisp.n.

Distribution. China (Hainan), Vietnam.

\section{Qiongthela bawang sp. n.}

http://zoobank.org/154A4D7B-7EF4-411C-8AAD-EE1952264EB5

Fig. 1

Holotype. Female (XUX-2011-001), Bawangling, Changiiang County, Hainan Province, China, $19.04^{\circ} \mathrm{N}, 109.09^{\circ} \mathrm{E}, 657 \mathrm{~m}$, collected 19 June 2011 by D. Li, F. Liu, M. Kuntner and X. Xu, deposited at CBEE, College of Life Sciences, Hubei University, Wuhan, China.

Paratypes. 2 females and 2 juveniles (XUX-2012-(094-097)), Bawangling Nature Reserve, Bawangling, Changjiang County, Hainan Province, China, $19.24^{\circ} \mathrm{N}$, $109.38^{\circ} \mathrm{E}, 462 \mathrm{~m}$, collected $19-20$ July 2012 by D. Li, F. Liu and X. Xu; 1 female (XUX-2014-012) collected at the same locality, $19.03^{\circ} \mathrm{N}, 109.10^{\circ} \mathrm{E}, 711 \mathrm{~m}$, collected 23 March 2014 by F. Liu and C. Xu.

Etymology. The species epithet, a noun in apposition, refers to the type locality. 


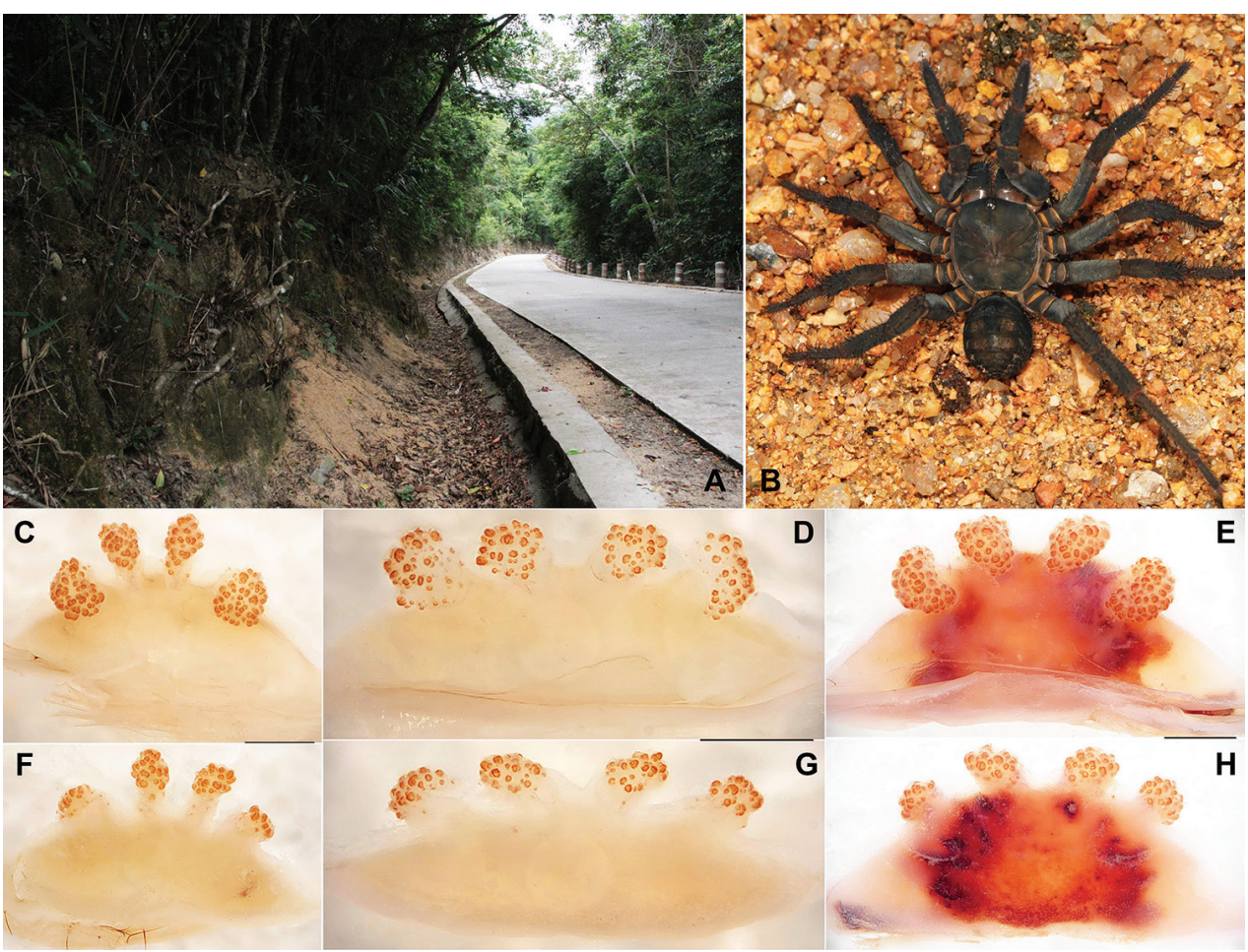

Figure I. Macrohabitat, general somatic morphology and genital anatomy of Qiongthela bawang sp. n. A Macrohabitat of Qiongthela bawang sp. n. at the type locality B Female (XUX-2012-097) C-E vulva dorsal view F-H vulva ventral view C, F (XUX-2014-012) D, G (XUX-2012-097) E, H (XUX-2011-001). Scale bars: $0.5 \mathrm{~mm}$.

Diagnosis. Females of the new species differ from Q. jianfeng sp. n. by the two pairs of receptacular clusters along the anterior margin of bursa copulatrix, which are visible in both dorsal and ventral views (Fig. 1C-H). It can be distinguished from $Q$. baishensis and $Q$. nui by its receptacular clusters with similar size or the middle pair being slightly smaller than the lateral pair. It also differs from all other Qiongthela species in Hainan by its receptacular clusters, all of which have short genital stalks (Fig. 1C$\mathrm{H})$. The male is unknown. To facilitate future identification of the species, we provided the DNA barcode for the holotype (XUX-2011-001), which is available on GenBank (Genbank accession code KP229897).

Description. Female (Fig. 1B). Carapace dark brown; opisthosoma darker than carapace; sternum narrow; a few long pointed hairs running over ocular mound in a longitudinal row; chelicerae robust with promargin of cheliceral groove containing 9-10 denticles of variable size; legs with strong hairs and spines; opisthosoma with 12 tergites, close to each other, the fifth largest; seven spinnerets. Measurements: BL 15.75-30.35, CL 8.37-15.95, CW 7.88-12.50, OL 7.42-16.50, OW 5.75-15.50; ALE $>$ PLE > PME > AME; palp $24.10(8.25+4.35+5.15+6.35)$, leg I $25.80(8.45+4.75$ 
$+5.05+5.00+2.55)$, leg II $24.70(7.65+4.50+4.85+5.00+2.70)$, leg III 26.80 $(7.85+4.80+4.70+6.10+3.35)$, leg IV $37.80(11.00+5.85+6.95+9.45+4.55)$.

Female genitalia. Two pairs of receptacular clusters along the anterior margin of bursa copulatrix, similar size or the middle pair slightly smaller, with short genital stalks (Fig. 1C-D)

Distribution. Hainan (Bawangling), China

\section{Qiongthela jianfeng sp. n.}

http://zoobank.org/91D190C7-2C68-4C7A-B957-D6E89E68AF4E

Fig. 2

Holotype. Male (XUX-2014-005), Jianfeng National Forest Park, Jianfeng Town, Ledong County, Hainan Province, China; $18.70^{\circ} \mathrm{N}, 108.84^{\circ} \mathrm{E}, 508 \mathrm{~m}$, collected 20 March 2014 by F. Liu and C. Xu, deposited at CBEE, College of Life Sciences, Hubei University, Wuhan, China.

Paratypes. One female (XUX-2014-002) and one male (XUX-2014-004, matured 2 August 2014 at CBEE, College of Life Sciences, Hubei University) collected at the same locality, 20 March 2014, by F. Liu and C. Xu; 1 male (XUX-2012-107) collected at the same locality, $18.70^{\circ} \mathrm{N}, 108.84^{\circ} \mathrm{E}, 500 \mathrm{~m}$, collected 22 July 2012 by D. Li, F. Liu and X. Xu; 1 female (XUX-2012-098) and 1 male (XUX-2012-100, matured 10 October 2012 at CBEE, College of Life Sciences, Hubei University) collected at the Forest Research Station, Jianfeng Town, Ledong County, Hainan Province, China, $18.70^{\circ} \mathrm{N}, 108.78^{\circ} \mathrm{E}, 145 \mathrm{~m}$, collected 21 July 2012 by D. Li, F. Liu and X. Xu; 1 female (XUX-2014-008) collected at the same locality, collected 21 March 2014 by F. Liu and C. Xu.

Etymology. The species epithet, a noun in apposition, refers to the type locality.

Diagnosis. Males of the new species differ from all other Qiongthela species by the semioval apophysis at the basal conductor (Fig. 2N, O), from Q. wuzhi sp. n. by a similar rectangular, rather than the basal angle between the two apophyses of tegulum more than $90^{\circ}$ (Fig. 2L), by the distal part of contrategulum with two edges, the inner one being dentate and the outer one being sharp (Fig. 2L, M), and by the distal edge of embolus slightly curved (Fig. 2M). Females of the new species differ from all other Qiongthela species by the receptaculuar clusters located slightly on the dorsal wall of the bursa copulatrix, especially the lateral pair being indistinct from the dorsal view (Fig. 2D-I). The DNA barcode of the paratype (XUX-2012-107) is available on GenBank (Genbank accession code KP229838) for future identification. The DNA barcodes of the holotype (XUX-2014-005) and paratype (XUX-2012-107) are identical (the K2P distance between the two sequences is zero).

Description. Male (Fig. 2C). Carapace dark; opisthosoma darker than carapace; sternum narrow; a few long pointed hairs running over ocular mound in a longitudinal row; chelicerae robust with promargin of cheliceral groove containing nine denticles of variable size; legs with strong hairs and spines; opisthosoma with 12 tergites, close 


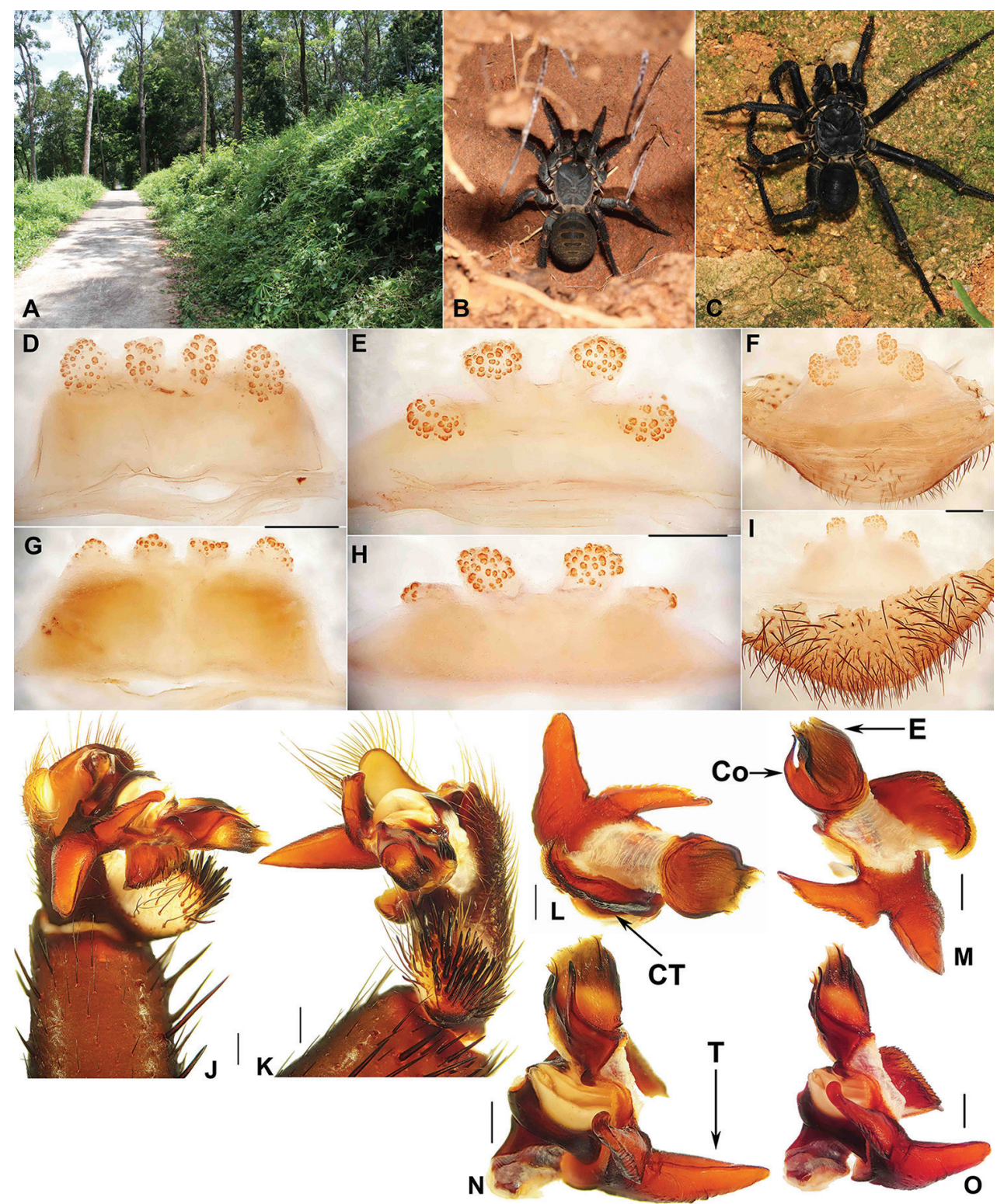

Figure 2. Macrohabitat, general somatic morphology and genital anatomy of Qiongthela jianfeng sp. n. A Macrohabitat of Qiongthela jianfeng sp. n. at the Forest Research Station B Female (XUX-2012-098) C male (XUX-2012-107) D-F vulva dorsal view G-I vulva ventral view J palp ventral view $\mathbf{K}$ palp retrolateral view L-O palp distal view D, G (XUX-2014-009) E, H (XUX-2014-002) F, I (XUX-2012-098) J-K (XUX-2014-004) L-O (XUX-2014-005). Scale bars: 0.5 mm.

to each other, the fifth largest; seven spinnerets. Measurements: BL 17.91-22.00, CL 7.35-12.2,0 CW 7.18-11.00, OL 10.35-10.00, OW 9.07-7.70; ALE > PLE > PME > AME; leg I $37.00(10.40+4.90+8.10+9.60+4.00)$, leg II $36.95(10.20+4.75+$ 
$7.50+10.30+4.20)$, leg III $36.90(9.70+4.80+6.80+11.00+4.60)$, leg IV 46.80 $(12.20+5.30+9.40+14.20+5.70)$.

Palp. The bulb of four specimens of the new species all relatively distorted. Prolateral side of paracymbium unpigmented and unsclerotised, many setae situated at the tip of paracymbium (Fig. 2J, K). Contrategulum with a proximally irregular dentate edge and two distal edges, the inner one dentate (Fig. 2L, M), the outer one sharp (Fig. 2L, M). Tegulum with a long, pointed, distally directed marginal apophysis with a smooth edge, a proximally directed terminal apophysis narrowing to a slightly bent apex, and the dorsal side of terimal apophysis with dentate edge (Fig. 2L, M). Conductor situated ventro-proximal part of embolus, fused with embolus at the basal portion, distal free, narrowing to a slightly hooked apex (Fig. 2M-O). Embolus largely sclerotised, with a wide, flat opening, curved distal edge (Fig. 2M).

Female (Fig. 2B). Colouration of carapace and opisthosoma similar to or lighter than male according to the age of specimens; chelicerae robust with promargin of cheliceral groove with 9-10 strong denticles of variable size; legs furnished with strong hairs and spines; opisthosoma with 12 tergites, similar to male; seven spinnerets. Measurements: BL 14.75-20.00, CL 7.25-11.56, CW 6.20-10.04, OL 7.30-12.00, OW 5.60-9.50; ALE $>$ PLE > PME > AME; palp $12.96(4.55+2.25+2.80+3.36)$, leg I $15.47(4.96+2.65+$ $2.91+3.25+1.70)$, leg II $14.40(4.55+2.30+2.65+3.25+1.65)$, leg III $15.50(4.35$ $+2.75+2.60+3.75+2.05)$, leg IV $22.51(6.36+3.20+3.95+6.15+2.85)$.

Female genitalia. Two pairs of receptacular clusters located slightly on the dorsal wall of the bursa copulatrix, especial the lateral pair indistinct in dorsal view, each receptacular cluster similar size, with or without a genital stalk (Fig. 2D-I).

Distribution. Hainan (Jianfeng), China

\section{Qiongthela yini sp. n.}

http://zoobank.org/DD0398BF-75CE-4ED2-9B8A-38615935DD78

Fig. 3

Holotype. Female (XUX-2012-106, matured 18 July 2013 at CBEE, College of Life Sciences, Hubei University), Jianfengling National Forest Park, Jianfeng Town, Ledong County, Hainan Province, China, $18.70^{\circ} \mathrm{N}, 108.86^{\circ} \mathrm{E}, 764 \mathrm{~m}$, collected 22 July 2012 by D. Li, F. Liu and X. Xu, deposited at CBEE, College of Life Sciences, Hubei University, Wuhan, China.

Etymology. The specific epithet honors the arachnologist Changmin Yin, a pioneering liphistiid specialist in China.

Diagnosis. The female of the new species differs from the other Qiongthela species by the receptacular clusters with less granula, the middle pair larger than the lateral pair, the middle pair along the anterior margin of bursa copulatrix, and the lateral pair located on the dorsal wall of the bursa copulatrix (Fig. 3B-C). The male is unknown. The DNA barcode of the holotype (XUX-2012-106) is available on GenBank (Genbank accession code KP229895) for facilitating future identification of the species. 


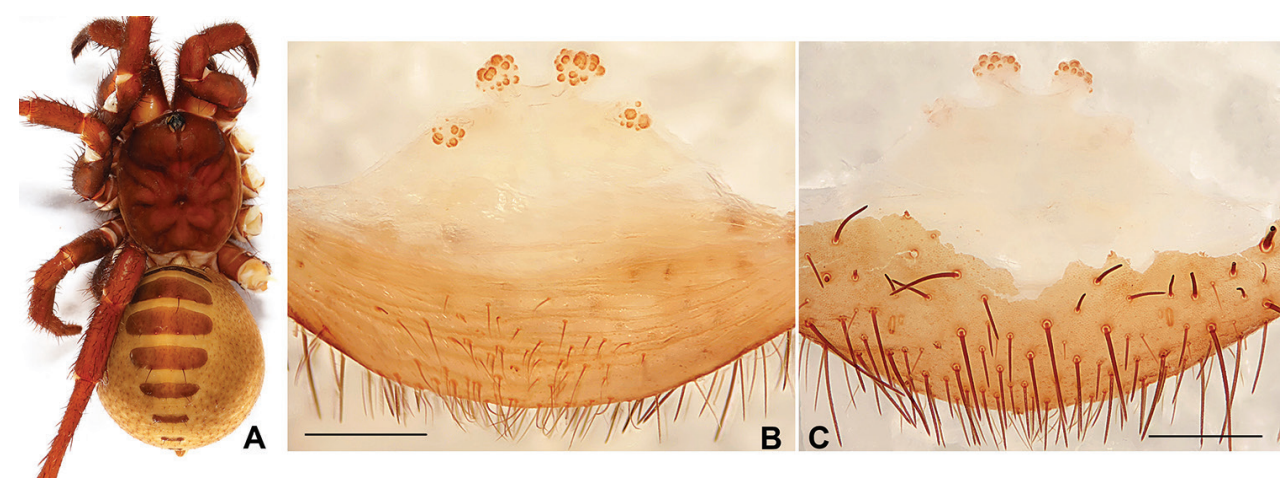

Figure 3. General somatic morphology and genital anatomy of Qiongthela yini sp. n. B Female (XUX-2012-106) B vulva dorsal view C vulva ventral view. Scale bars: $0.5 \mathrm{~mm}$.

Description. Female (Fig. 3A). Carapace reddish brown in alcohol; opisthosoma light brown; sternum narrow, much longer than wide; a few long pointed hairs running over ocular mound in a longitudinal row; chelicerae robust with promargin of cheliceral groove with ten strong denticles of variable size; legs furnished with strong hairs and spines; opisthosoma with 12 tergites, separated from each other, the first 2-7 larger than others and the fifth largest; seven spinnerets. Measurements: BL 20.50, CL 8.00, CW 7.21, OL 12.40, OW 10.00; ALE > PLE > PME > AME; palp $13.54(4.70+$ $2.43+2.80+3.61), \operatorname{leg}$ I $15.90(5.10+2.80+3.11+3.20+1.69)$, leg II $15.35(4.70$ $+2.80+2.79+3.41+1.65)$, leg III $13.85(4.35+2.10+2.60+3.00+1.80)$, leg IV $23.71(7.00+3.35+4.18+6.30+2.88)$.

Female genitalia. The middle receptacular clusters along the anterior margin of bursa copulatrix, the lateral pair located on the dorsal wall of the bursa copulatrix, the middle pair larger than the lateral pair, and the middle pair with short genital stalks (Fig. 3B).

Distribution. Hainan (Jianfeng), China.

Remarks. This new species was found at the Jianfengling National Forest Park as some specimens of $Q$. jianfeng sp. n., but at a higher altitude compared with the latter.

\section{Qiongthela wuzhi sp. n.}

http://zoobank.org/19D93B63-0BD5-446F-8E4C-E2058521B02A

Fig. 4

Holotype. Male (XUX-2012-109, matured 4 October 2012 at CBEE, College of Life Sciences, Hubei University), Yongxun Village, Shuiman Town, Wuzhishan City, Hainan Province, China, $18.90^{\circ} \mathrm{N}, 109.63^{\circ} \mathrm{E}, 551 \mathrm{~m}$, collected 25 July 2012 by D. Li, F. Liu and $\mathrm{X}$. Xu, deposited at CBEE, College of Life Sciences, Hubei University, Wuhan, China.

Paratypes. One female (XUX-2012-108) collected at the same locality, collected 25 July 2012 by D. Li, F. Liu and X. Xu. 


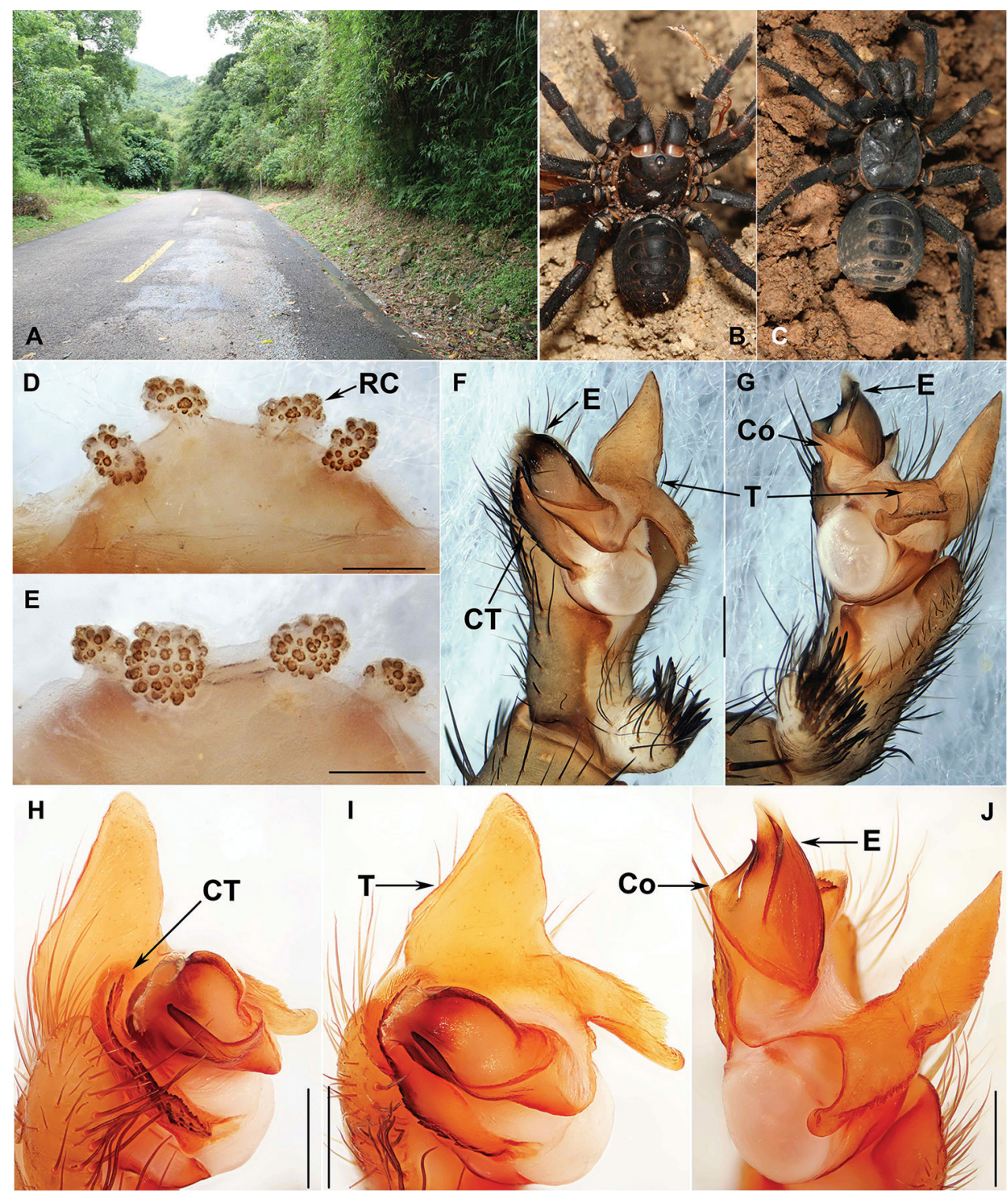

Figure 4. Macrohabitat, general somatic morphology and genital anatomy of Qiongthela wuzhi sp. n. A Macrohabitat of Qiongthela wuzhi sp. n. at the type locality B female (XUX-2012-108) C male (XUX2012-109) D vulva dorsal view E vulva ventral view $\mathbf{F}$ palp prolateral view $\mathbf{G}$ palp retrolateral view H-J palp distal view. Scale bars: $0.5 \mathrm{~mm}$.

Etymology. The species epithet, a noun in apposition, refers to the type locality.

Diagnosis. Male of this new species differs from Q. jianfeng sp. n. by the contrategulum with three distal edges (Fig. $4 \mathrm{H}, \mathrm{J}$ ), the basal angle between the two apophyses of tegulum more than $90^{\circ}$ (Fig. 4I), and the smooth distal margin of embolus (Fig. 4I). 
It differs from Q. baishensis, Q. australi,s and Q. nui by three distal margins of contrategulum (Fig. 4H, J). Females of this new species can be distinguished from the other species of Qiongthela by the receptacular clusters with very short genital stalks, and from $Q$. bawang sp. n. by the irregular shapes of receptacular clusters (Fig. 4D, E). The DNA barcode of the paratype (XUX-2012-108) is available on GenBank (Genbank accession code KP229812) for future identification. The DNA barcodes of the holotype (XUX-2012-109) and paratype (XUX-2012-108) are identical (the K2P distance between the two sequences is zero).

Description. Male (holotype) (Fig. 4C). Carapace black; opisthosoma light brown; sternum narrow, nearly twice as long as wide; a few long pointed hairs running over ocular mound in a longitudinal row; chelicerae robust with promargin of cheliceral groove with eight denticles of variable size; legs furnished with strong hairs and spines; opisthosoma with 12 tergites, the first 2-7 close to each other and larger than others; seven spinnerets. Measurements: BL 14.75, CL 6.20, CW 6.45, OL 9.65, OW 7.45; ALE > PLE > PME > AME; leg I $17.71(5.03+2.52+3.86+4.50+1.80)$, leg II $17.88(5.00+2.35+$ $3.67+4.48+2.38)$, leg III $18.87(4.65+2.55+3.52+5.45+2.70)$, leg IV miss.

Palp. Prolateral side of paracymbium unpigmented and unsclerotised, many setae situated at the tip of paracymbium (Fig. 4F. G). Contrategulum with a proximally irregular dentate edge and three distal edges, the inner one dentate, very short, the middle one dentate, running down to the proximally irregular dentate edge of contrategulum, the outer one sharp, fused with the inner one at the middle portion of the middle edge (Fig. 4H-J). Tegulum with a wide base, pointed, distally directed marginal apophysis with a sharp edge, with a proximally directed terminal apophysis narrowing to a slightly bent apex, and the dorsal side of terminal apophysis with dentate edge (Fig. 4F, G, I, J). Embolus largely sclerotised, with a wide, flat opening, and a sharp distal edge (Fig. 4F-J).

Female (Fig. 4B). Colouration of carapace and opisthosoma reddish dark; female similar to male except larger than male in size; chelicerae robust with promargin of cheliceral groove with 10 strong denticles of variable size; legs furnished with strong hairs and spines; opisthosoma with 12 tergites, tergites larger than that of male; 7 spinnerets. Measurements: BL 20.35, CL 10.35, CW 8.55, OL 11.25, OW 8.65; ALE > PLE > PME > AME; palp $18.28(6.35+3.18+4.00+4.75)$, leg I $21.27(7.05+3.75$ $+4.12+4.48+1.87), \operatorname{leg}$ II $20.06(6.67+3.27+3.62+4.45+2.05), \operatorname{leg}$ III 20.82 $(6.18+3.75+3.35+5.07+2.47)$, leg IV $30.54(8.90+4.50+5.51+8.13+3.50)$.

Female genitalia. The two pairs of receptacular clusters along the anterior margin of bursa copulatrix, irregular receptacular clusters with very short genital stalks (Fig. 4D, E).

Distribution. Hainan (Wuzhishan), China.

\section{Acknowledgements}

This study was supported by the grants to X.X. from the National Natural Sciences Foundation of China (NSFC-31601850) and the Hunan Provincial Natural Science 
Foundation of China (2017JJ3202), and the grants to D.L. from the Singapore Ministry of Education (MOE) AcRF Tier 1 grant (R-154-000-638-112) and the National Natural Sciences Foundation of China (NSFC-31272324). We thank Ingi Agnarsson and Hirotsugu Ono for their insightful comments on our manuscript. We also thank Hongzhe Li, Xiaoyan Wang, and Chen Xu for their help in the field and the staff of the Centre for Behavioural Ecology and Evolution (CBEE), Hubei University and Hunan Normal University for all their help and support throughout this study.

\section{References}

Ono H (2002) Occurrence of a heptatheline spider (Araneae, Liphistiidae) in Lam Dong province, Vietnam. Bulletin of the National Museum of Nature and Science Tokyo (A) 28: $119-122$.

Schwendinger PJ, Ono H (2011) On two Heptathela species from southern Vietnam, with a discussion of copulatory organs and systematics of the Liphistiidae (Araneae: Mesothelae). Revue Suisse de Zoologie 118: 599-637. https://doi.org/10.5962/bhl.part.117818

Xu X, Liu FX, Cheng R-C, Chen J, Xu X, Zhang ZS, Ono H, Pham DS, Norma-Rashid Y, Arnedo MA, Kuntner M, Li D (2015a) Extant primitively segmented spiders have recently diversified from an ancient lineage. Proceedings of the Royal Society B: Biological Sciences 282: 20142486. https://doi.org/10.1098/rspb.2014.2486

Xu X, Liu FX, Chen J, Ono H, Li D, Kuntner M (2015b) A genus level taxonomic revision of primitively segmented spiders (Mesothelae: Liphistiidae). ZooKeys 488: 121-151. https:// doi.org/10.3897/zookeys.488.8726

Xu X, Liu FX, Chen J, Li D, Kuntner M (2015c) Integrative taxonomy of the primitively segmented spider genus Ganthela (Araneae: Mesothelae: Liphistiidae) - DNA barcoding gap agrees with morphology. Zoological Journal of the Linnean Society 175: 288-306. https:// doi.org/10.1111/zoj.12280

Xu X, Liu FX, Chen J, Ono H, Agnarsson I, Li D, Kuntner M (2016) Pre-Pleistocene geological events shaping diversification and distribution of primitively segmented spiders on East Asian Margins. Journal of Biogeograpy 43: 1004-1019. https://doi.org/10.1111/jbi.12687

Xu X, Liu FX, Ono H, Chen J, Kuntner M, Li D (2017) Targeted sampling in Ryukyus facilitates species delimitation of the primitively segmented spider genus Ryuthela (Araneae: Mesothelae: Liphistiidae). Zoological Journal of the Linnean Society. https://doi. org/10.1093/zoolinnean/zlx024

World Spider Catalog (2017) World spider catalog. Natural History Museum Bern. http:// wsc.nmbe.ch [version 18.5, accessed on 1 November 2017] 